\title{
Influencia Del Nivel De Instrucción En La Capacidad Emprendedora
}

\author{
Mario Beltrán Apolo \\ Andrés Pacheco Molina \\ Bill Serrano Orellana \\ Luis Brito-Gaona \\ Universidad Técnica de Machala - Ecuador
}

doi: 10.19044/esj.2016.v12n25p131 URL:http://dx.doi.org/10.19044/esj.2016.v12n25p131

\begin{abstract}
This research analyzed the influence of the level of education in entrepreneurship. The study included 982 participants in Machala-Ecuador. A survey with 20 questions to help determine the entrepreneurial ability to then provide a statistical analysis through a linear regression model, analysis where the dependent variable is quantitative and the independent variable is dichotomous. The results indicate a significant relationship between the level of education and entrepreneurship, as well as in each of the five dimensions involved. It is concluded that the ability for individuals to undertake entrepreneurship is proportional to the level of education they posses. These findings may be useful for both the general population as well as public or private institutions which employ mechanisms to promote both management and development of enterprises.
\end{abstract}

Keywords: Entrepreneurship, entrepreneur, level of instruction

\section{Resumen}

En el presente trabajo se analiza la influencia del nivel de instrucción sobre la capacidad emprendedora. Para ello se tomó una muestra de 982 habitantes de la ciudad de Machala - Ecuador, en la cual se aplicó una encuesta conformada por un cuestionario de 20 preguntas que determinan la capacidad emprendedora para luego dar paso a un análisis estadístico mediante un modelo de regresión lineal donde la variable dependiente es cuantitativa y la independiente de naturaleza dicotómica. Los resultados muestran una relación altamente significativa entre el nivel de instrucción y la capacidad emprendedora, así como en cada una de las cinco dimensiones que la conforman. Se concluye que la capacidad para emprender de los individuos es proporcional al nivel de instrucción que poseen. Estos hallazgos pueden ser 
útiles tanto para la población en general como para instituciones públicas o privadas que empleen mecanismos que promuevan la gestión y el desarrollo de emprendimientos.

Palabras claves: Emprendimiento, emprendedor, nivel de instrucción

\section{Introducción}

En la actualidad la creación de emprendimientos se ha convertido en una actividad socioeconómica con un vasto potencial generador de empleo e ingresos, que paralelamente al empleo con relación de dependencia son los ejes centrales de la actividad laboral actual. Nos encontramos en un contexto empresarial donde se evidencia una tendencia creciente relacionada con la creación de negocios por parte de individuos que se ven motivados por distintas razones, tales como: el desempleo, el subempleo, la necesidad de independencia, entre otros. El fenómeno emprendedor es constantemente objeto de análisis para determinar las motivaciones que tienen los emprendedores para empezar su camino empresarial, pero la búsqueda debe ser constante debido los incesantes y en ocasiones acelerados cambios que sufre el entorno socioeconómico de un determinado sector que se ven afectados tanto por factores internos como externos. Es importante conocer los aspectos que están directamente relacionados con la generación de emprendimientos y el vínculo que pueda existir con otros factores que deriven en la generación de nueva y relevante información que permita encontrar soluciones eficientes.

Según un estudio del Global Entrepreneurship Monitor (GEM) en 2014, Ecuador es el país con mayor índice de actividad emprendedora temprana (TEA) de la región con un leve descenso con respecto a los 5 años anteriores. Lo que nos indica la importancia que empieza a tener el emprendimiento y su contribución con el desarrollo económico en el país. Dentro del estudio proporcionado por el GEM se identificó que el 39,4\% de los emprendedores solo completo sus estudios primarios, mientras que el $25,2 \%$ los estudios secundarios y finalmente un 13,9\% completó sus estudios terciarios. Sin embargo, en el estudio no se pretendió relacionar al nivel de instrucción con el resto de variables para identificar algún tipo de relación causal. La incorporación del emprendimiento en el contexto económico del país provoca la generación de nueva información de la que surgen hipótesis que necesitan ser respondidas.

La creación de emprendimientos actualmente está siendo apoyada en buena medida por el estado mediante programas de capacitación y programas inclusivos que brindan un capital semilla. Pero es necesario que los organismos gubernamentales canalicen de una manera más eficiente los recursos mediante el conocimiento que tengan de los emprendedores, de sus 
habilidades y comportamiento. Puesto que, según Rodrigo, Parra y García (20014) hay que saber identificar las actitudes ya que estas definen realmente al perfil del emprendedor. Identificar las características de los emprendedores y relacionarlas con distintas variables es sin dudas un foco generador de conocimiento. Mientras más se conozca los distintos rasgos propios del emprendedor será más fácil fomentar la generación de ideas (Zárate , 2013). Ideas que, para Duarte (2007) nos proporcionan oportunidades de mejora constante. Si bien el emprendimiento forma parte de desarrollo económico del país, conocerlo más a fondo permitirá fomentar iniciativas que le permitan crecer y evolucionar para romper ciertas barreras que impidan su expansión y del mismo modo obtener un beneficio colectivo. Dicho beneficio radica en el impacto bilateral que resulta del aprendizaje proporcionado por la generación de nuevo conocimiento a partir del análisis de las variables abordadas anteriormente y considerando desde el punto de vista socioeconómico donde los individuos buscan una oportunidad de progreso con la generación de emprendimientos y el estado obtiene retribuciones gracias a los ingresos y la generación de empleos que provoca el fenómeno emprendedor. Por lo tanto el crecimiento socioeconómico de un sector se verá reflejado notoriamente en la mejora de la calidad de vida de la sociedad. Y así como la información es importante para organismos gubernamentales lo es para la sociedad y el buen uso que se puedan brindar, ya sea utilizándolo como un soporte de información para el desarrollo de emprendimientos o aportando conocimiento relacionado al mismo, que le permita expandirse y encontrar más información que sea de utilidad para la colectividad.

En el presente trabajo el enfoque se centraliza en analizar la relación existente entre la capacidad emprendedora y el nivel de formación educativa o de instrucción del individuo. Dicho de otro modo, si la formación académica de las personas influye en su capacidad para generar emprendimientos. Y así, generar nuevo conocimiento que contribuya a la praxis académica diaria y con especial énfasis en ser objeto de análisis. Mediante el uso de un instrumento de medición utilizado por de la Universidad Nacional Mayor de San Marcos por el investigador Oscar Tinoco Gómez (Tinoco, 2008). Se analizarán aspectos claves englobados en cinco dimensiones como el conocimiento de sí mismo, la visión de futuro, la motivación de logro, planeación y persuasión de los individuos, para luego ser analizados desde el punto de vista asociado al nivel de instrucción de los individuos, para al final poder descifrar el enigma que rodea a la hipótesis planteada y determinar si el nivel de instrucción juega un rol determinante en la capacidad emprendedora.

\section{Marco Teórico}

Muchos autores resaltan la importancia del emprendimiento en el desarrollo económico de una sociedad, tal es el caso de Matiz (2009) quien 
menciona que el crecimiento socioeconómico de individuos y países está directamente relacionado con gestión del emprendimiento. Siendo el emprendimiento un proceso transformador de recursos cuyo fin es satisfacer necesidades (Petit, 2007). Del mismo modo Serrano Bedia, Pérez Pérez, Palma Ruiz y López Fernández (2016) mencionan que el emprendimiento como oportunidad de desarrollo sustentable para economías domésticas se convierte en un factor de alto impacto socioeconómico. La constante búsqueda del mejoramiento de la calidad de vida lleva a las sociedades a cambiar sus procesos y dar lugar a nuevos conocimientos creando así una cultura de emprendimiento. Desde estas perspectivas el emprendimiento como buscador de resultados padece de un abanico de posibilidades de desarrollo para individuos y conglomerados que buscan nuevas oportunidades de progreso.

Una cultura emprendedora vista como gestor de desarrollo económico de la sociedad también está inmersa en el desarrollo personal de los individuos, ya que según Marulanda Valencia, Montoya Restrepo y Vélez Restrepo (2014) desarrolla en los individuos habilidades tales como la comunicación, planificación, liderazgo, trabajo en equipo, creatividad, entre otras. Las características o aptitudes que identifican a un emprendedor resulta significativo a nivel de comprensión del comportamiento emprendedor (Castellanos, Chávez, \& Jiménez, 2003). Y gracias a este desarrollo de habilidades Fernández, Alegre, y Chiva (2012) acotan que el emprendimiento fortalece los alcances de los individuos creando un ciclo constante de conocimiento.

Por su parte Castro, Saavedra y Camarena (2015) nos ayudan a conocer al emprendedor y resaltan que es imperioso diferenciar claramente a un emprendedor de un empresario y comprender que el empresario busca maximizar el capital y el emprendedor se inclina hacia los resultados. Son múltiples las formas en que se intenta descifrar ciertos paradigmas que contribuyan a la comprensión de lo implica el termino emprendedor. De acuerdo con Orrego (2008) el emprendedor aparte de tener muy en claro el panorama en que se desenvuelve responde de manera positiva con nuevas ideas junto a un constante sentido de iniciativa. Además Barrera (2012) propone que es importante incluir dentro de estos aspectos la responsabilidad social de sus acciones y su gestión de manera particular desde tres puntos de vista diferentes tanto desde lo económico así como lo social y ambiental. El emprendedor busca constantemente innovar mediante un agudo sentido de la propensión al riesgo para mejorar sus condiciones de vida y obtener un mayor beneficio.

Paralelamente con el emprendedor, la capacidad emprendedora se convierte en el factor decisivo que impulsa el desarrollo de un emprendimiento y por lo tanto en el centro de análisis de la investigación. Referirse a la capacidad emprendedora resulta desafiante ya que no cuenta con una 
definición estable y aceptada mundialmente. Así por ejemplo Moreno (2002) relaciona a la capacidad emprendedora con el desarrollo de habilidades que deriven en la solución de problemas. Habilidades que, para Rusque (2005) están relacionadas con aspectos psicológicos tales como la creatividad, toma de riesgos, necesidad de logro, independencia y autonomía, y la determinación. Y estas habilidades que de manera conjunta, derivan en una connotación ligada al desarrollo empresarial (Duarte \& Ruiz, 2009). Puesto que, analizar la intencionalidad de crear una empresa resulta desafiante en muchos aspectos. Para Restrepo (2013) uno de los aspectos críticos y tal vez el principal es el cambio del entorno y su ardua adaptabilidad. Olaya (2008) Añade que el entorno se puede ver afectado principalmente por factores socioeconómicos, políticos y ambientales. Sin embargo la superación de estos desafíos es en esencia una base del espíritu emprendedor. Por lo tanto, dada la importancia y consecuentemente un análisis de las características o aspectos que distinguen al emprendedor permite identificar entre un emprendedor de éxito del resto (Quintana, 2001).

La capacidad emprendedora puede ser analizada entorno a cinco aspectos principales como son: conocimiento de sí mismo y autoconfianza, visión de futuro, motivación de logro, planificación y persuasión (Tinoco, 2008). Mismos que derivan en una secuencia de aspectos analizados entre sí que permiten dar una valoración proporcional a la validez de cada aspecto.

Conocimiento de sí mismo: Para Martinez y Carmona (2009) el conocimiento de si mismo implica en tener la confianza suficiente en los conocimientos propios para tomar decisiones determinantes. La confianza provee la seguridad necesaria para disminuir la incertidumbre (Naranjo, Jiménnez, \& Sanz, 2012). Entonces, se puede decir que, mientras mayor sea el conocimiento de si mismo, existirá menos incertidumbre y mayor seguridad, para una toma de decisiones eficiente.

Visión de Futuro: Las aspiraciones, anhelos e ideales que se quieran alcanzar o concretar componen una visión de futuro que nos es más que fijarse donde se quiere llegar en un futuro cercano. Espíritu (2011) acota que para que una visión de futuro sea viable deben plantearse objetivos alcanzables. Puesto que, para que los objetivos sean alcanzables se debe establecer una clara y meticulosa diferencia entre el exceso de optimismo y la realidad palpable.

Motivación de Logro: Se refiere al deseo o la necesidad de conseguir objetivos. Sin embargo para Morán y Menezes (2016) va más allá de solo alcanzar objetivos, puesto que incluye a un perspicaz sentido de la mejora, es decir, conseguir más y mejores cosas. También señala una estrecha relación entre la motivación de logro y el desarrollo económico de un sector, pues mientras mayor sea el deseo por lograr resultados óptimos mayor será la probabilidad de percibir mejoramientos importantes. 
Planificación: Salazar \& Romero (2006) definen a la planificación como una herramienta que sirve como guía para alcanzar objetivos y que está directamente relacionada con el éxito desde un entorno social. También recalca que la planificación debe ser ejecutada de manera metodológica evitando usar a la intuición como único recurso. En ese sentido Cárdenas (2013) señala que la metodología de la planificación se relaciona en la asignación adecuada recursos. Entonces, el aseguramiento de una planificación exitosa se deberá en gran medida a la sinergia creada por los recursos implicados en la misma, a su desempeño en cada uno de los grupos de interés y a los resultados obtenidos.

Persuasión: Comúnmente la persuasión es asociada a la habilidad de lograr que una persona cambie de decisión. En el entorno empresarial la persuasión puede ser determinante si se pretende tener las situaciones a favor (Ripollés \& Blesa, 2006). Puesto que, según Ponjuán (1999) usar la persuasión adecuadamente en situaciones de conflicto puede solucionar los problemas de una manera satisfactoria para las partes involucradas.

El nivel de instrucción y la capacidad emprendedora ya han sido relacionados en la intencionalidad de crear una empresa. Tal y como lo mencionan Galindo y Méndez (2011) indicando que la formación profesional en algunos países por más alta que esta sea inclina a los individuos a conseguir empleo en lugar de crear una empresa. Optar por empleo como primera opción es producto de la incertidumbre que genera la presión sobre los individuos en torno a definir un futuro estable (Marulanda, Montoya, \& Veéez, 2014). Y es que, el nivel de instrucción resulta significativo según García, Martínez y Fernández (2010) quienes exponen que la experiencia previa y la formación recibida son factores significativos para la creación o dirección de una empresa.

La importancia del nivel de instrucción ha sido desde siempre una preocupación más por una sociedad que busca oportunidades que les permitan mejorar su calidad de vida. Oportunidades que serán más alcanzables mientras mejor preparación exista (Matiz, 2009). Para Orozco y Chavarro (2008) la valoración de una buena preparación educativa es para la mayoría de las culturas vista como símbolo de estatus y reconocimiento social. Mozas (2013) añade que el aprendizaje también se ve fortalecido por la experiencia reunida a través de experiencias similares. Puesto que la experiencia también es parte de la esencia del emprendedor. Para Álvarez y Urbano (2013) poseer experiencia previa facilita la adaptación al entorno. Entonces, podemos decir que la capacidad emprendedora se ve influenciada por el resultado de una combinación entre la experiencia y el conocimiento muy bien acoplados entre sí.

De distintas maneras de ha incentivado en exceso a que las personas emprendan sus ideas de negocio sin tomar en cuenta la importancia de tener 
una formación específica (Matiz B, 2006). Misma que involucrada en el proceso emprendedor consigue desarrollar aptitudes y modificar el comportamiento con una visión hacia procesos más eficientes (De La Fuente, Vera, \& Cardelle, 2012). Ya que forma parte de los rasgos distintivos del emprendedor que se ven involucrados en el crecimiento empresarial (Hurtado Ayala, Escandón, \& Hurtado, 2014). Lo que lleva a la conclusión más predecible, que para Sepulveda y Gutiérrez (2016) consiste en que emprender sin conocimiento específico o formación profesional no es garantía de éxito.

Existe un amplio debate sobre el nivel de instrucción entorno al emprendimiento, ya que muchos investigadores revelan que un gran número de personas han conseguido llevar a cabo sus ideas de negocio con simple conocimiento empírico y se cuestiona sobre lo que se necesita saber para disminuir el factor riesgo y ser un emprendedor de éxito. Sin embargo, en los últimos años se han desarrollado varias investigaciones en torno a la capacidad emprendedora como método de medición relacionando distintas variables psicológicas de los individuos involucrados donde se evidencia el uso superficial del nivel de instrucción como aspecto relacionado a la capacidad emprendedora mas no como una variable determinante, ni que se intente buscar incidencia con el resto de factores y saber si su influencia podría ser un agente causal. Por lo tanto, con lo ya antes mencionado se plantea la siguiente hipótesis:

“La capacidad emprendedora es determinada por el nivel de instrucción?"

\section{Metodología}

\section{Datos y características de la muestra}

La información utilizada en el presente trabajo investigativo es producto de la implementación del instrumento validado por (Flores, 2003), aplicada a los habitantes de la ciudad de Machala, se detalla la ficha técnica en la Tabla 1.

Tabla 1: Ficha Técnica del Estudio

\begin{tabular}{cc}
\hline Unidad de Análisis: & Individuos \\
Entorno Geográfico: & Machala, El Oro, Ecuador \\
Universo de la Población: & Habitantes de la ciudad de Machala \\
Tamaño de la Población: & 276.669 \\
Tamaño de la muestra: & 982 \\
Fecha de Elaboración: & 2016 \\
\hline
\end{tabular}

Nota: Elaboración propia 


\section{Descripción de las variables}

Variable Dependiente

Para el análisis se empleó a la capacidad emprendedora como variable dependiente que permitió determinar si los individuos cuentan con las suficientes competencias que le permitan gestionar un emprendimiento. Esta variable gira entorno a cinco dimensiones: conocimiento de sí mismo y autoconfianza, visión de futuro, motivación de logro, planificación y persuasión, y que posteriormente fueron evaluadas por medio de un análisis estadístico comprendido por pruebas de regresión lineal. Para el análisis se asignaron valores en las siguientes proporciones: nunca (0), casi nunca (1), en ocasiones (2), con frecuencia (3), casi siempre (4) y siempre (5). La calificación máxima a obtener es 100 .

\section{Variable Independiente}

Para este estudio la variable independiente es el nivel de instrucción y se estableció en una escala de cuatro niveles: primaria, secundaria, superior y postgrado. Con una asignación de valores de 0 a 3 respectivamente.

\section{Metodología de análisis}

El presente trabajo pretende demostrar el grado de influencia entre la capacidad emprendedora y el nivel de instrucción. Inicialmente se encara un análisis psicológico del individuo para determinar su capacidad emprendedora para luego relacionarlo con su nivel de instrucción, del mismo modo se analiza el nivel de instrucción con cada una de las dimensiones de la capacidad emprendedora. La encuesta para determinar la capacidad emprendedora fue aplicada en el año 2016. El análisis de datos se lo realizó a través de la aplicación de modelos de regresión lineal por medio del software Stata para Windows.

Se utilizó un modelo desde una óptica cuantitativa donde la variable dependiente es una variable con escala de razón y la independiente es de naturaleza dicotómica. Para determinar el nivel de la capacidad emprendedora se utiliza la escala de calificación propuesta en la Tabla 2.

Tabla 2: Escala de calificación del instrumento

\begin{tabular}{cc}
\hline Puntaje & Calificación \\
\hline De1 a 4 & Muy bajo \\
De 5 a 8 & Bajo \\
De 9 a 12 & Mediano \\
De 13 a 16 & Alto \\
De 17 a 20 & Muy alto
\end{tabular}

Nota: tomada de Tinoco (2008) 


\section{Hipótesis} instrucción

H1: La capacidad emprendedora es determinada por el nivel de

H2: Existen diferencias significativas entre las dimensiones de la capacidad emprendedora y los niveles de instrucción involucrados.

\section{Resultados}

Los resultados obtenidos (tabla 3) revelan una relación directa y altamente significativa $(\mathrm{p}<0,01)$ entre el nivel de instrucción y la capacidad emprendedora .Quienes tienen mayor capacidad emprendedora son aquellos individuos que registran un nivel universitario, mientras que los individuos que menor capacidad emprendedora tienen son aquellos que registran un nivel de instrucción primario, confirmando así lo propuesto en la primera hipótesis.

Tabla 3: Resultados del análisis de regresión

\begin{tabular}{|c|c|}
\hline & Coef \\
\hline \multicolumn{2}{|c|}{ Capacidad emprendedora } \\
\hline Secundaria & $\begin{array}{c}8.973128^{* *} \\
(4,69)\end{array}$ \\
\hline Superior & $\begin{array}{c}14.41155^{* * *} \\
(7,63)\end{array}$ \\
\hline Postgrado & $\begin{array}{c}13.02081 \text { ** } \\
(5,55)\end{array}$ \\
\hline Primaria & 64.46154 \\
\hline R-Squared & 0,0702 \\
\hline $\mathrm{F}(3,978)$ & $24,63 * *$ \\
\hline \multicolumn{2}{|c|}{ Conocimiento de sí mismo } \\
\hline Secundaria & $\begin{array}{c}2.373744 * * \\
\quad(5.00)\end{array}$ \\
\hline Superior & $\begin{array}{c}3.635112 * * \\
\quad(7.77)\end{array}$ \\
\hline Postgrado & $\begin{array}{c}3.887783^{* *} \\
(6.68)\end{array}$ \\
\hline Primaria & 11.87692 \\
\hline R-Squared & 0,0744 \\
\hline $\mathrm{F}(3,978)$ & $26,19 * *$ \\
\hline \multicolumn{2}{|c|}{ Visión de futuro } \\
\hline Secundaria & $\begin{array}{c}1.306256^{* *} \\
(2.86)\end{array}$ \\
\hline Superior & $\begin{array}{c}2.281737 * * * \\
\quad(5.06)\end{array}$ \\
\hline Postgrado & $\begin{array}{c}2.888688^{* * *} \\
(5.15)\end{array}$ \\
\hline Primaria & 13.12308 \\
\hline R-Squared & 0,0430 \\
\hline $\mathrm{F}(3,978)$ & $14,65 * *$ \\
\hline \multicolumn{2}{|c|}{ Motivación de logro } \\
\hline Secundaria & $1.597128 * *$ \\
\hline
\end{tabular}




\begin{tabular}{cc}
\hline Superior & $(3.71)$ \\
& $2.271065^{* *}$ \\
Postgrado & $(5.35)$ \\
& $1.056109^{*}$ \\
Primaria & $(2.00)$ \\
R-Squared & 14.06154 \\
F(3, 978) & 0,0358 \\
Planificación & $12,12^{* *}$ \\
Secundaria & $2.137231^{* *}$ \\
& $(4.73)$ \\
Superior & $3.10271^{* *}$ \\
Postgrado & $(6.96)$ \\
Primaria & $2.239819^{* *}$ \\
R-Squared & $(4.04)$ \\
F(3,978) & 13.03077 \\
Persuasión & 0,0535 \\
Secundaria & $18,44^{* *}$ \\
& \\
Superior & $1.558769^{* *}$ \\
Postgrado & $(3.30)$ \\
Primaria & $3.120922^{* *}$ \\
R-Squared & $(6.69)$ \\
F(3,978) & $2.948416^{* *}$ \\
& $(5.08)$ \\
& 12.36923 \\
& 0,0687 \\
& $24,04 *$ \\
\hline
\end{tabular}

Nota: el número superior de la celda es el coeficiente, los números entre paréntesis son estadísticos; $* * \mathrm{p}<0,01 ; * \mathrm{p}<0,05$.

Para el análisis de las cinco dimensiones de la capacidad emprendedora se comparó cada uno de los coeficientes de los niveles de instrucción con la constante que representa al nivel de instrucción primario. Según los resultados obtenidos tanto en el factor de conocimiento de sí mismo como en el factor de visión de futuro, quienes mayor puntaje obtuvieron son los individuos que registran un nivel de instrucción de postgrado. Mientras que los factores de motivación de logro, planificación y persuasión quienes mayor puntaje obtuvieron son los individuos que registran un nivel de instrucción de superior. Concluyendo que mientras mayor sea el nivel de instrucción mayor será el dominio de las dimensiones de la capacidad emprendedora.

Dentro del análisis de las cinco dimensiones de la capacidad emprendedora se determina que existen diferencias entre las dimensiones de la capacidad emprendedora y los niveles de instrucción involucrados, ya que la relación es altamente significativa $(\mathrm{p}<0,01)$, es decir, que los niveles de instrucción más altos poseen los puntajes más altos en cada una de las dimensiones. Validando así la segunda hipótesis. 
La escala de calificación (tabla 5) determina que los individuos con nivel de instrucción superior poseen una capacidad emprendedora de nivel alto. Los puntajes de cada una de las dimensiones fueron establecidos según resultado obtenido en la tabla 4 donde se establece la media de cada una de las dimensiones de la capacidad emprendedora, relacionadas con cada uno de los niveles de instrucción, para luego ser comparados según la escala de calificación de la tabla 2 y finalmente resumidos en la tabla 5 donde se promedian los valores correspondientes a las medias y se establece el nivel de la capacidad correspondiente a cada nivel de instrucción, cuyos resultados son altamente significativos, como lo muestra la tabla 3.

Tabla 4: Puntaje total obtenido según dimensión evaluada

\begin{tabular}{|c|c|c|c|c|c|c|}
\hline \multirow{3}{*}{$\begin{array}{l}\text { Conocimiento } \\
\text { de sí mismo }\end{array}$} & & Primaria & Secundaria & Superior & Postgrado & Total \\
\hline & mean & 11.877 & 14.251 & 15.512 & 15.765 & 14.812 \\
\hline & ds & 3.769 & 3.320 & 3.427 & 4.631 & 3.663 \\
\hline \multirow{4}{*}{$\begin{array}{l}\text { Visión de } \\
\text { futuro }\end{array}$} & $\mathrm{cv}$ & .317 & .233 & .221 & .294 & .247 \\
\hline & mean & 13.123 & 14.429 & 15.405 & 16.012 & 14.934 \\
\hline & ds & 3.352 & 3.693 & 3.223 & 3.014 & 3.472 \\
\hline & $\mathrm{cv}$ & .255 & .256 & .209 & .188 & .233 \\
\hline \multirow{3}{*}{$\begin{array}{l}\text { Motivación de } \\
\text { logro }\end{array}$} & mean & 14.062 & 15.659 & 16.333 & 15.118 & 15.820 \\
\hline & ds & 4.111 & 3.071 & 3.018 & 3.877 & 3.256 \\
\hline & $\mathrm{cv}$ & .292 & .196 & .185 & .256 & .206 \\
\hline \multirow[t]{3}{*}{ Planificación } & mean & 13.030 & 15.168 & 16.133 & 15.271 & 15.485 \\
\hline & ds & 4.458 & 3.560 & 2.989 & 3.396 & 3.451 \\
\hline & $\mathrm{cv}$ & .342 & .235 & .185 & .222 & .223 \\
\hline \multirow[t]{3}{*}{ Persuasión } & mean & 12.369 & 13.928 & 15.490 & 15.318 & 14.672 \\
\hline & ds & 4.414 & 3.692 & 3.329 & 2.920 & 3.642 \\
\hline & $\mathrm{cv}$ & .357 & .265 & .215 & .191 & .248 \\
\hline
\end{tabular}

Nota: Elaboración propia

Tabla 5: Puntaje total obtenido (capacidad emprendedora) según nivel de instrucción

\begin{tabular}{cccccc}
\hline $\mathrm{Ni}$ & $\mathrm{cv}$ & $\mathrm{Sd}$ & Mean & Promedio & Nivel \\
\hline Primaria & .272 & 17.508 & 64.462 & 12.892 & mediano \\
Secundaria & .195 & 14.335 & 73.435 & 14.687 & Alto \\
Superior & .170 & 13.391 & 78.873 & 15.775 & Alto \\
Postgrado & .201 & 15.581 & 77.482 & 15.496 & Alto \\
Total & .195 & 14.757 & 75.722 & & \\
\hline \multicolumn{7}{c}{ Elaboración propia } & & &
\end{tabular}

\section{Conclusiones}

La presente investigación se desarrolla en un contexto socioeconómico en constante desarrollo, donde la creación de emprendimientos está en auge y los estudios sobre emprendimiento son insuficientes para tener una base sólida de conocimiento, para el cual se propuso el objetivo que consistía en 
determinar si el nivel de instrucción es un factor lo suficientemente influyente en la capacidad emprendedora.

Los resultados muestran cómo el nivel de instrucción influye en la capacidad emprendedora de una manera clara y específica, al notar que los niveles más altos de instrucción son aquellos que poseen mayor trascendencia, concluyendo que, los individuos que cuentan con un nivel de instrucción superior son quienes tienen un mejor desarrollo de sus competencias y los convierte en lo suficientemente aptos para la gestión de emprendimientos, con una capacidad emprendedora de nivel alto.

Los resultados en cada una de las dimensiones de la capacidad emprendedora revelan que existen diferencias significativas entre los niveles de instrucción involucrados, puesto que los niveles de instrucción superior y postgrado son los más determinantes en cada una las cinco dimensiones de la capacidad emprendedora, mientras que los niveles inferiores como secundaria y primaria no son relevantes.

Los resultados del análisis estadístico son de naturaleza progresiva, lo que nos lleva a concluir que, a mayor nivel de instrucción mayor será la capacidad emprendedora. Todo parece indicar que la influencia de los conocimientos adquiridos por los individuos incrementa sus competencias y disminuyen sus limitaciones haciéndolos más propensos al autoempleo.

\section{References:}

Alegre-Vidal, J., Chiva-Gomez, R., \& Fernandez, A. (2012). Orientación emprendedora, capacidad de aprendizaje organizativo y desempeño innovador. Journal Of Technology Management \& Innovation, 7(2), 157-169. Alvarez, C., \& Urbano, D. (2013). Diversidad cultural y emprendimiento. Revista de Ciencias Sociales, 19(1), 154-169.

Alvarez, C., \& Urbano, D. (2013). Diversidad cultural y emprendimiento. Revista de Ciencias Sociales, 19(1), 154-169.

Barrera Malpica, S. P. (2012). Análisis del campo del emprendimiento desde un estudio de casos. Ciencias Estratégicas, 20(27), 133-145.

Cárdenas, L. (2013). Metodologías de planificación y los nuevos enfoques de sustentabilidad. Urbano, 16(27), 9-22.

Castellanos, O. F., Chávez, R. D., \& Jiménez, C. N. (2003). Propuesta de formación en liderazgo y emprendimiento. Innovar Revista de Ciencias Administrativas y Sociales(22), 145-156.

Castro, A. B., Saavedra García, M. L., \& Camarena Adame, M. E. (2015). Hacia una comprensión de los conceptos de emprendedores. Suma de negocios, 6(13), 98-107.

De La Fuente, J., Vera, M., \& Cardelle, M. (2012). Aportaciones de la psicología de la innovación y del emprendimeinto a la educación, en la 
sociedad del conocimiento. Electronic Journal of research in educational psychology, 10(28), 941-966.

Duarte, F. (2007). Emprendimiento, empresa y crecimiento empresarial. Contabilidad y negocios, 2(3), 46-56.

Duarte, T., \& Ruiz, M. (2009). Emprendimiento, una opción para el desarrollo. Scientia Et Technica, 15(43), 326-331.

Espíritu, R. (2011). Análisis de la intención emprendedora en estudiantes universitarios a través de los rasgos de personalidad. MULTICIENCIAS, 11(1), 67-75.

Galindo Martín, M. A., \& Méndez Picazo, M. T. (2011). La actividad emprendedora y competitividad: factores que inciden sobre los emprendedores. Papeles de Europa, 22, 61-75.

García Ramos, C., Martínez Campillo, A., \& Fernández Gago, R. (2010). Caracteristicas del emprendedor influyentes en el proceso de creación empresarial y en el éxito esperadp. Revista Europea de dirección y economía de la empresa, 19(2), 31-48.

Hurtado Ayala, A., Escandón, D., \& Hurtado, A. (2014). Factores que influyen en el desarrollo exportador de las pymes en Colombia. Estudios Gerenciales, 30, 172-183.

Martinez, F., \& Carmona, M. (2009). Aproximaciones al concepto de competencias emprendedoras: valor social e implicaciones educativas. Revista Iberoamericana sobre Calidad, Eficiencia y Cambio en Educación, 7(3), 8298.

Marulanda Valencia, F. A., Montoya Restrepo, I. A., \& Vélez Restrepo, J. M. (2014). Teorias motivacionales en el estudio del emprendimiento. Pensamiento \& Gestión(36), 206-238.

Marulanda, F., Montoya, I., \& Veéez, J. (2014). Aportes teóricos y empíricos al estudio del emprendedor. Cuadernos de Administración, 30(51).

Matiz B, F. J. (2006). Emprendimiento como un pilar para la competitividad de las naciones. Escuela de administración de negocios(57), 119-129.

Matiz, F. J. (2009). Investigación en emprendimiento, un reto para la construcción. Revista EAN(66), 169-182.

Morán, C., \& Menezes, E. (2016). La motivación de logro como impluso creador de bienestar: su relación con los cinco grandes factores de la personalidad. International Journal of Developmental And Educational Psychology, 2(1), 31-40.

Moreno, J. (2002). Capacidad emprendedora en la educacion superior. Balance de la experiencia del servicio de atención al emprendedor(SAE) en la universidad metropolitana. ANALES, 2(2), 149-160.

Mozas, A. (2013). Emprendimiento y economía social. CIRIEC(78), 7-9.

Naranjo, J., Jiménnez, D., \& Sanz, R. (2012). ¿Es la cultura organizativa un determinante de la innovación en la empresa? CEDE, 15, 63-72. 
Olaya, A. (2008). Economía de la innovación y del cambio tecnológico: una aproximación teorica desde el pensamiento Schumpeteriano. Ciencias Estratégicas, 16(20), 237-246.

Orozco, L., \& Chavarro, D. (2008). Universidad y emprendimiento. Hallazgos (10), 65-97.

Orrego, C. I. (2008). La dimensión humanda del emprendimiento. Ciencias Estratégicas, 16(20), 225-235.

Petit, E. (2007). La gerencia emprendedora innovadora como catalizador del emprendimiento económico. Revista de Ciencias Sociales RCS, 13(3), 495506.

Ponjuán, G. (1999). El éxito de la gestión o la gestión del éxito. Anales de Documentación(2), 39-47.

Quintana, C. (2001). Dimensiones del exito de las empresas emprendedoras. Investigaciones europeas de dirección y economía de las empresas, 7(2), 139158.

Restrepo, C. (2013). Aproximación a la gestión de la innovación: un enfoque conceptual. Ciencias Estratégicas, 22(30), 257-266.

Ripollés, M., \& Blesa, A. (2006). Redes personales del empresario y orientación emprendedora en las nuevas empresas. Cuadernos de Economía y Dirección de la Empresa(26), 73-94.

Rodrigo, J., Parra, G., \& García, P. (20014). Efectividad de la orientación emprendedora : el papel del capital social y las capacidades. Investigaciones Europeas de Dirección y Economía de las Empresas, 20(3), 1-9.

Rodríguez Ramírez, A. (2009). Nuevas perspectivas para entender. Pensamiento \& gestión, 26, 94-119.

Rusque, A. M. (2005). Capacidad emprendedora y capital social. Revista venezolana de análisis de coyuntura, 11(2), 189-202.

Salazar, D., \& Romero, G. (2006). Planificación. ¿Éxito Gerencial? Multiciencias, 6(1), 1-17.

Sepulveda Rivas, C. I., \& Gutiérrez Walter, R. (2016). Sostenibilidad de los emprendimientos. Análisis de los factores determinantes. Revista Venezolana de Gerencia, 21(73), 33-49.

Serrano Bedia, A. M., Pérez Pérez, M., Palma Ruiz, M., \& López Fernández, M. C. (2016). Emprendimiento: Visión actual como disciplina de investigación.Un análisis de los números publicados durante 2011 y 2013. Estudios Gerenciales, 32, 89-95.

Tinoco, O. (2008). Medición de la capacidad emprendedora de ingresantes a la facultad de ingenieria industrial de la UNMSM. Industrial Data, 11(2), 1823.

Zárate, R. (2013). Emprendimiento: Diferentes aproximaciones. Escuela de Administración de Negocios(74), 176-178. 